\title{
On the Existence and Stability of Standing Waves for 2-Coupled Nonlinear Fractional Schrödinger System
}

\author{
Xiuyan Sha, Huanmin Ge, and Jie Xin \\ School of Mathematics and Statistics Science, Ludong University, Yantai 264025, China \\ Correspondence should be addressed to Jie Xin; fdxinjie@sina.com
}

Received 25 September 2015; Accepted 1 December 2015

Academic Editor: Seenith Sivasundaram

Copyright ( 2015 Xiuyan Sha et al. This is an open access article distributed under the Creative Commons Attribution License, which permits unrestricted use, distribution, and reproduction in any medium, provided the original work is properly cited.

\begin{abstract}
We study a system of 2-coupled nonlinear fractional Schrödinger equations. Firstly, we construct constrained minimization problem to the system. Next, we prove the existence of standing waves for the system by using the concentration-compactness and commutator estimates method. Lastly, we also consider the set of minimizers of the constrained minimization problem. We prove that it is a stable set for initial value of the problem; that is, a solution to the system with initial value which is near the set will remain near it for all time.
\end{abstract}

\section{Introduction}

In the recent years, more and more researchers study the application of fractional calculus and fractional integrodifferential equations in physics and other areas (see [1-4]). The concept of fractional calculus is firstly put forward by Leibniz as a generalization of standard calculus. Afterwards several kinds of definitions of them have been established such as Riemann-Liouville fractional derivative, Caputo fractional derivative, and Weyl fractional derivative (see $[5,6])$. We all know that a variety of fractional calculus is brought in inspired by standard calculus, but the calculation laws of fractional calculus are much different (see $[5,7])$. To avoid the complicated fractional calculus, in this paper we are only concerned with the fractional Laplacian operator.

Here we study the existence and stability of standing waves for the following system of 2-coupled nonlinear fractional Schrödinger equations:

$$
\begin{array}{r}
i \psi_{1 t}+(-\Delta)^{\alpha} \psi_{1}=\gamma\left(\left|\psi_{1}\right|^{p-2}+\left|\psi_{1}\right|^{p / 2-2}\left|\psi_{2}\right|^{p / 2}\right) \psi_{1}, \\
x \in \mathbb{R}^{N}, \\
i \psi_{2 t}+(-\Delta)^{\alpha} \psi_{2}=\gamma\left(\left|\psi_{2}\right|^{p-2}+\left|\psi_{2}\right|^{p / 2-2}\left|\psi_{1}\right|^{p / 2}\right) \psi_{2}, \\
x \in \mathbb{R}^{N},
\end{array}
$$

where $i^{2}=-1, N \geqslant 2,2<p<2+4 \alpha / N, \gamma>0,0<\alpha<1$, and $(-\Delta)^{\alpha}$ is a pseudo-differential operator which is defined by $\left(\widehat{-\Delta)^{\alpha} \psi_{i}}(\xi)=|\xi|^{2 \alpha} \widehat{\psi}_{i}(\xi)\right.$, the Fourier transform $F \psi_{i}(\xi)=$ $\widehat{\psi}_{i}(\xi)=\left(1 /(2 \pi)^{N / 2}\right) \int_{\mathbb{R}^{N}} \psi_{i}(x) e^{-i \xi \cdot x} d x$.

It is well known that the coupled nonlinear Schrödinger equations play an important role in describing nonrelativistic quantum mechanical behavior. In particular, the fractional Schrödinger equations can describe better some real physical phenomenon. Schrödinger type equation(s) is first derived by Feynman and Hibbs, applying path integrals over Brownian paths in [8]. In [9-11], Laskin showed the path integral over Lévy-like quantum mechanical paths approaches to a generalization of quantum mechanics. As the path integral over Brownian trajectories, it leads to the standard Schrödinger equations. And as the path integral over Lévy-like quantum mechanical paths, it leads to the fractional Schrödinger equation. Recently, there are some researchers studying fractional Schrödinger equation(s) and its physical applications (see [12-14]). The research of the existence and stability of standing waves for the nonlinear Schrödinger equations arises in various fields of physics such as plasma physics, constructive field theory, and nonlinear optics (see [15-22]). There are many papers about this topic for the standard Schrödinger equations with different nonlinear terms such as $[17,19,20]$. There are some results concerned with the existence and stability of standing waves for the nonlinear 
fractional Schrödinger equations in [21, 22]. The author [23] obtained the global solution for a class of systems of fractional nonlinear Schrödinger equations with boundary condition. However, there are few results about the standing waves of fractional nonlinear Schrödinger equations (1). In the present paper, we consider the existence and stability of standing waves for the system of 2-coupled nonlinear fractional Schrödinger equations.

The rest of this paper is arranged as follows. In Section 2, we recall some basic definitions and introduce preparation results. In Section 3, we give the main results and proof of them.

\section{Preliminaries}

In this section, we firstly introduce the constrained minimization problem in order to study the existence and stability of standing waves for (1). We know that the standing waves of (1) have the special form $\left(\psi_{1}(x, t), \psi_{2}(x, t)\right)=$ $\left(e^{i \omega_{1} t} u_{1}(x), e^{i \omega_{2} t} u_{2}(x)\right)$, where $\omega_{i} \in \mathbb{R}(i=1,2)$. Then we only need to find $\omega_{1}, \omega_{2}, u_{1}(x), u_{2}(x)$ satisfying the following equations to study the existence and stability of standing waves of (1):

$$
\begin{array}{r}
(-\Delta)^{\alpha} u_{1}-\omega_{1} u_{1}=\gamma\left(\left|u_{1}\right|^{p-2}+\left|u_{1}\right|^{p / 2-2}\left|u_{2}\right|^{p / 2}\right) u_{1}, \\
x \in \mathbb{R}^{N}, \\
(-\Delta)^{\alpha} u_{2}-\omega_{2} u_{2}=\gamma\left(\left|u_{2}\right|^{p-2}+\left|u_{2}\right|^{p / 2-2}\left|u_{1}\right|^{p / 2}\right) u_{2}, \\
x \in \mathbb{R}^{N},
\end{array}
$$

where $u_{1}, u_{2}$ are complex value functions.

By the variational method, we know that in order to study the existence of solution for (2) we need to consider the following constrained minimization problem:

$$
\begin{aligned}
I_{q} & :=\inf \left\{I\left(u_{1}, u_{2}\right) \mid\left(u_{1}, u_{2}\right) \in H^{\alpha}\left(\mathbb{R}^{N}\right)\right. \\
& \left.\times H^{\alpha}\left(\mathbb{R}^{N}\right), M\left(u_{1}, u_{2}\right)=q, q>0\right\},
\end{aligned}
$$

where

$$
\begin{aligned}
& I\left(u_{1}, u_{2}\right) \\
& =\frac{1}{2} \int_{\mathbb{R}^{N}}\left(\left|(-\Delta)^{\alpha / 2} u_{1}\right|^{2}+\left|(-\Delta)^{\alpha / 2} u_{2}\right|^{2}\right) d x \\
& \quad-\int_{\mathbb{R}^{N}} F\left(x, u_{1}, u_{2}\right) d x, \\
& M\left(u_{1}, u_{2}\right)=\int_{\mathbb{R}^{N}}\left(\left|u_{1}\right|^{2}+\left|u_{2}\right|^{2}\right) d x, \\
& \partial_{u_{1}} F\left(x, u_{1}, u_{2}\right)=\gamma\left(\left|u_{1}\right|^{p-2}+\left|u_{1}\right|^{p / 2-2}\left|u_{2}\right|^{p / 2}\right) u_{1}, \\
& \partial_{u_{2}} F\left(x, u_{1}, u_{2}\right)=\gamma\left(\left|u_{2}\right|^{p-2}+\left|u_{2}\right|^{p / 2-2}\left|u_{1}\right|^{p / 2}\right) u_{2} .
\end{aligned}
$$

And $H^{\alpha}\left(\mathbb{R}^{N}\right)$ is the fractional order Sobolev space. We denote

$$
\begin{aligned}
G_{q} & :=\left\{\left(u_{1}, u_{2}\right) \mid\left(u_{1}, u_{2}\right) \in H^{\alpha}\left(\mathbb{R}^{N}\right)\right. \\
& \left.\times H^{\alpha}\left(\mathbb{R}^{N}\right), M\left(u_{1}, u_{2}\right)=q, I\left(u_{1}, u_{2}\right)=I_{q}\right\}
\end{aligned}
$$

as the set of minimizers of the problem $I_{q}$.

Next we recall some basic definitions. Define the fractional order Sobolev space

$$
\begin{aligned}
& H^{\alpha}\left(\mathbb{R}^{N}\right)=\{u \mid u \\
& \left.\quad \in L^{2}\left(\mathbb{R}^{N} ; \mathbb{C}\right), F^{-1}\left[\left(1+|\xi|^{2}\right)^{\alpha / 2} F u\right] \in L^{2}\left(\mathbb{R}^{N}\right)\right\}
\end{aligned}
$$

with the norm

$$
\|\cdot\|_{\alpha, 2}=\left|F^{-1}\left(1+|\xi|^{2}\right)^{\alpha / 2} F u\right|_{2} .
$$

Denote the product space $H^{\alpha}\left(\mathbb{R}^{N}\right) \times H^{\alpha}\left(\mathbb{R}^{N}\right)=\left\{\left(u_{1}, u_{2}\right) \mid\right.$ $\left.u_{1}, u_{2} \in H^{\alpha}\left(\mathbb{R}^{N}\right)\right\}$ with the norm $\left\|\left(u_{1}, u_{2}\right)\right\|=\left(\left\|u_{1}\right\|_{\alpha, 2}^{2}+\right.$ $\left.\left\|u_{2}\right\|_{\alpha, 2}^{2}\right)^{1 / 2}$. Throughout this paper, we denote the norm of $L_{\text {per }}^{p}\left(\mathbb{R}^{N}\right)$ by $|\cdot|_{p}$ for all $1 \leqslant p \leqslant \infty$. Denote the set $B(y, r)=\left\{x\left|x, y \in \mathbb{R}^{N}, r>0,\right| x-y \mid<r\right\}$. For simplicity and convenience, the letter $C$ will represent a constant, which may be different from one to others. $C(\cdot, \cdot)$, for example, represents the constant $C$ which can be expressed by the parameters appearing in the braces.

Lastly, we give the following some lemmas, which will be used in our paper. For more detail, we can see [21, 23, 24].

Lemma 1. If $0<\alpha<1, x \in \mathbb{R}^{N}$, and $u \in S$, $S$ representing the Schwartz class, then the fractional Laplacian $(-\Delta)^{\alpha}$ of $u$ is also expressed for the formula

$$
(-\Delta)^{\alpha} u(x)=C(N, \alpha) P . V \cdot \int_{\mathbb{R}^{N}} \frac{u(x)-u(y)}{|x-y|^{N+2 \alpha}} d x,
$$

where P.V. means the Cauchy principal value on the integral and $C(N, \alpha)$ is some positive normalization constant.

Lemma 2. For $0<\alpha<1$, there are two properties with $H^{\alpha}\left(\mathbb{R}^{N}\right)$ :

(i) The norm $\|\cdot\|_{\alpha, 2}$ of $H^{\alpha}\left(\mathbb{R}^{N}\right)$ is equivalent to $\|\cdot\|=$ $\left|F^{-1}\left[\left(1+|\xi|^{\alpha}\right) F \cdot\right]\right|_{2}$.

(ii) $\forall u \in H^{\alpha}\left(\mathbb{R}^{N}\right), c\|u\|_{W^{\alpha, 2}\left(\mathbb{R}^{N}\right)} \leqslant\|u\| \leqslant C\|u\|_{W^{\alpha, 2}\left(\mathbb{R}^{N}\right)}$,

which implies $H^{\alpha}\left(\mathbb{R}^{N}\right)=W^{\alpha, 2}\left(\mathbb{R}^{N}\right)$, where $W^{\alpha, 2}\left(\mathbb{R}^{N}\right)$ is defined by the method of trace interpolation (see chapter 7 of [24]) and its norm is given by $\|u\|_{W^{\alpha, 2}\left(\mathbb{R}^{N)}\right)} c$ and $C$ are positive constants.

Remark 3. In the following paper, we denote the norm $\|\cdot\|_{\alpha, 2}$ of $H^{\alpha}\left(\mathbb{R}^{N}\right)$ by $\|\cdot\|$.

Lemma 4 (see [24]). For $0<\alpha<1,2 \leqslant p_{1} \leqslant 2 N /(N-2 \alpha)$, $H^{\alpha}\left(\mathbb{R}^{N}\right) \hookrightarrow L^{p_{1}}\left(\mathbb{R}^{N}\right)$ and $W^{\alpha, 2}(B(y, r)) \hookrightarrow L^{p_{1}}(B(y, r))$. 
Moreover, if $u_{n} \rightarrow u$ in $H^{\alpha}\left(\mathbb{R}^{N}\right)$, then $u_{n} \rightarrow u$ in $L_{\text {loc }}^{p}\left(\mathbb{R}^{N}\right)$, and $u_{n} \rightarrow u$ almost everywhere in $\mathbb{R}^{N}$, where $2 \leqslant p<$ $2 N /(N-2 \alpha)$.

Lemma 5. If $\left\{\left(u_{1 n}, u_{2 n}\right)\right\}$ is bounded in $H^{\alpha}\left(\mathbb{R}^{N}\right) \times H^{\alpha}\left(\mathbb{R}^{N}\right)$ and $\lim _{n \rightarrow+\infty} \sup _{y \in \mathbb{R}^{N}} \int_{B(y, r)}\left(\left|u_{1 n}\right|^{2}+\left|u_{2 n}\right|^{2}\right) d x=0$ for some $r>0$, then $\lim _{n \rightarrow+\infty}\left(u_{1 n}, u_{2 n}\right)=(0,0)$ in $L^{p}\left(\mathbb{R}^{N}\right) \times L^{p}\left(\mathbb{R}^{N}\right)$ for $2<p<2 N /(N-2 \alpha)$.

Remark 6. The proof of Lemma 5 is obtained easily according to Lemma 2.4 (see [21]), so we will omit it.

Lemma 7 (commutator estimates). If $0<\alpha<1, f, g \in S$, the Schwartz class, then

$$
\begin{aligned}
& \left|(-\Delta)^{\alpha / 2}(f g)-f(-\Delta)^{\alpha / 2} g\right|_{2} \\
& \quad \leqslant C\left(|\nabla f|_{\bar{p}_{1}}\left|(-\Delta)^{(\alpha-1) / 2} g\right|_{\bar{q}_{1}}+\left|(-\Delta)^{\alpha / 2} f\right|_{\bar{p}_{2}}|g|_{\bar{q}_{2}}\right),
\end{aligned}
$$

where $\bar{p}_{2}, \bar{q}_{1} \in[2,+\infty)$ satisfying $1 / \bar{p}_{1}+1 / \bar{q}_{1}=1 / \bar{p}_{2}+1 / \bar{q}_{2}=$ $1 / 2$.

In [23], the authors have obtained the existence and uniqueness of the global solution of a class of systems for fractional nonlinear Schrödinger equations with periodic boundary condition by using the Faedo-Galërkin method. In the proof of Theorem 4.1 in the references, only needing to let the period $2 \pi e_{i} \rightarrow+\infty$, we can obtain the following theorem.

Theorem 8. Let $0<\alpha<1$. If $\Psi_{0}=\left(\Psi_{10}, \Psi_{20}\right) \in H^{\alpha}\left(\mathbb{R}^{N}\right) \times$ $H^{\alpha}\left(\mathbb{R}^{N}\right)$ then there exists a global weak solution $\Psi(x, t)=$ $\left(\Psi_{1}(x, t), \Psi_{2}(x, t)\right) \in C\left(\mathbb{R}, H^{\alpha}\left(\mathbb{R}^{N}\right) \times H^{\alpha}\left(\mathbb{R}^{N}\right)\right)$ to the initial value problem of coupled nonlinear fractional Schrödinger equations (1) with initial date $\Psi_{10}=\Psi_{1}(x, 0), \Psi_{20}=\Psi_{2}(x, 0)$.

\section{Uniform Estimates of the Solution}

In the section, we consider coupled nonlinear equations (2). Since the scaling $u_{i \gamma}=\gamma^{1 /(p-2)} u_{i}(x)(i=1,2)$, we can transform (2) to the following equations:

$$
\begin{aligned}
& (-\Delta)^{\alpha} u_{1 \gamma}-\omega_{1} u_{1 \gamma} \\
& =\left(\left|u_{1 \gamma}\right|^{p-2}+\left|u_{1 \gamma}\right|^{p / 2-2}\left|u_{2 \gamma}\right|^{p / 2}\right) u_{1 \gamma}, \quad x \in \mathbb{R}^{N}, \\
& (-\Delta)^{\alpha} u_{2 \gamma}-\omega_{2} u_{2 \gamma} \\
& =\left(\left|u_{2 \gamma}\right|^{p-2}+\left|u_{1 \gamma}\right|^{p / 2}\left|u_{2 \gamma}\right|^{p / 2-2}\right) u_{2 \gamma}, \quad x \in \mathbb{R}^{N} .
\end{aligned}
$$

So we only consider coupled nonlinear equations (10). So we have

$$
F\left(x, u_{1}, u_{2}\right)=\frac{1}{p}\left(\left|u_{1}\right|^{p}+\left|u_{2}\right|^{p}\right)+\frac{2}{p}\left|u_{1}\right|^{p / 2}\left|u_{2}\right|^{p / 2} .
$$

For any $q>0$, let $\widetilde{I}_{q}:=I_{q}$ and $\widetilde{G}:=G_{q}$.
Under the case we have the following main results.

Theorem 9. Assuming $\left\{\left(u_{1 n}, u_{2 n}\right)\right\}$ is a minimizing sequence of problem $\widetilde{I_{q}}$, then there exists a sequence $\left\{y_{n}\right\} \in \mathbb{R}^{N}$ such that $\left\{\left(u_{1 n}\left(\cdot+y_{n}\right), u_{2 n}\left(\cdot+y_{n}\right)\right\}\right.$ contains a convergent subsequence in $H^{\alpha}\left(\mathbb{R}^{N}\right) \times H^{\alpha}\left(\mathbb{R}^{N}\right)$. In particular, there exists a minimizer for problem $\left(\widetilde{I}_{q}\right)$ (i.e., $\widetilde{G}_{q} \neq \phi$ ), and

$$
\lim _{n \rightarrow+\infty} \inf _{\left(g_{1}, g_{2}\right) \in \widetilde{G}}\left(\left\|u_{1 n}-g_{1}\right\|+\left\|u_{2 n}-g_{2}\right\|\right)=0 .
$$

Theorem 10. The set $\widetilde{G}_{q}$ is $H^{\alpha}\left(\mathbb{R}^{N}\right) \times H^{\alpha}\left(\mathbb{R}^{N}\right)$-stable with respect to (1); that is, $\forall \varepsilon>0$, there exists $\delta>0$ such that if $\left(u_{1}, u_{2}\right) \in C\left([0, T), H^{\alpha}\left(\mathbb{R}^{N}\right) \times H^{\alpha}\left(\mathbb{R}^{N}\right)\right)$ is a solution of $(1)$ with initial value $\left(u_{10}, u_{20}\right)$ satisfying

$$
\inf _{\left(g_{1}, g_{2}\right) \in \widetilde{G}_{q}}\left(\left\|u_{10}-g_{1}\right\|+\left\|u_{20}-g_{2}\right\|\right)<\delta,
$$

then, $\forall t \in[0, T)$,

$$
\inf _{\left(g_{1}, g_{2}\right) \in \widetilde{G}_{q}}\left(\left\|u_{1}(t, \cdot)-g_{1}\right\|+\left\|u_{2}(t, \cdot)-g_{2}\right\|\right)<\varepsilon .
$$

Before we give the proof of Theorems 9 and 10, we firstly prove the following some lemmas.

Lemma 11. For any $q>0$, one has the following:

(i) $-\infty<\widetilde{I}_{q}<0$,

(ii) every minimizing sequence problem $\widetilde{I}_{q}$ is bounded in $H^{\alpha}\left(\mathbb{R}^{N}\right) \times H^{\alpha}\left(\mathbb{R}^{N}\right)$. And for sufficiently large $n$, there exists a positive constant $\delta$ such that $\left|u_{1 n}\right|_{p}+\left|u_{2 n}\right|_{p} \geqslant \delta$.

Proof. (i) For given $\left(u_{1}, u_{2}\right) \in H^{\alpha}\left(\mathbb{R}^{N}\right) \times H^{\alpha}\left(\mathbb{R}^{N}\right),\left|u_{1}\right|_{2}^{2}+$ $\left|u_{2}\right|_{2}^{2}=q,\left|u_{1}\right|_{p}^{p}+\left|u_{2}\right|_{q}^{q} \neq 0$. And set $u_{i \theta}(x)=\theta^{1 / 2} u_{i}\left(\theta^{1 / N} x\right)(i=$ $1,2)$; then $\left|u_{1 \theta}\right|_{2}^{2}+\left|u_{2 \theta}\right|_{2}^{2}=\left|u_{1}\right|_{2}^{2}+\left|u_{2}\right|_{2}^{2}=q$.

By Lemma 1, we have that

$$
\begin{aligned}
\widetilde{I}\left(u_{1 \theta}, u_{2 \theta}\right) & \frac{1}{2} \theta^{2 \alpha / N} \int_{\mathbb{R}^{N}}\left(\left|(-\Delta)^{\alpha / 2} u_{1}\right|^{2}+\left|(-\Delta)^{\alpha / 2} u_{2}\right|^{2}\right) d x \\
& -\frac{1}{p} \theta^{p / 2-1} \int_{\mathbb{R}^{N}}\left(\left|u_{1}\right|^{p}+\left|u_{2}\right|^{p}\right) d x \\
& -\frac{2}{p} \theta^{p / 2-1} \int_{\mathbb{R}^{N}}\left|u_{1}\right|^{p / 2}\left|u_{2}\right|^{p / 2} d x \\
\leqslant & \frac{1}{2} \theta^{2 \alpha / N} \int_{\mathbb{R}^{N}}\left(\left|(-\Delta)^{\alpha / 2} u_{1}\right|^{2}+\left|(-\Delta)^{\alpha / 2} u_{2}\right|^{2}\right) d x \\
& -\frac{1}{p} \theta^{p / 2-1} \int_{\mathbb{R}^{N}}\left(\left|u_{1}\right|^{p}+\left|u_{1}\right|^{p}\right) d x .
\end{aligned}
$$

By $0<p / 2-1<2 \alpha / N$, there exists $\widetilde{I}\left(u_{1 \theta}, u_{2 \theta}\right)<0$ taking sufficiently small $\theta$. Therefore, we obtain that $\widetilde{I} \leqslant \widetilde{I}\left(u_{1 \theta}, u_{2 \theta}\right)<0$.

By the Hölder inequality and the Sobolev inequality, we can derive

$$
\left|u_{i}\right|_{p} \leqslant\left|u_{i}\right|_{2}^{(1-\lambda)}\left|u_{i}\right|_{2 N /(N-2 \alpha)}^{\lambda} \leqslant C\left|u_{i}\right|_{2}^{1-\lambda} \|\left. u_{i}\right|^{\lambda}
$$


where $p(1-\lambda) / 2+\lambda p /(2 N /(N-2 \alpha))=1$. Because of the Young inequality and $0<\lambda p<2$, for $\varepsilon>0$, from the above inequality we can derive that

$$
\left|u_{i}\right|_{p}^{p} \leqslant \varepsilon\left\|u_{i}\right\|^{2}+C(\varepsilon)\left|u_{i}\right|_{2}^{2 p(1-\lambda) /(2-\lambda p)}, \quad i=1,2 .
$$

So for $\left(u_{1}, u_{2}\right) \in H^{\alpha}\left(\mathbb{R}^{N}\right) \times H^{\alpha}\left(\mathbb{R}^{N}\right)$, setting $\left|u_{i}\right|_{2}^{2}=q_{i}(i=$ $1,2), q_{1}+q_{2}=q$ by the Young inequality and (17), we have that

$$
\begin{aligned}
\widetilde{I}\left(u_{1}, u_{2}\right) & \\
\geqslant & \frac{1}{2}\left(\left\|u_{1}\right\|^{2}+\left\|u_{2}\right\|^{2}\right)-\frac{1}{2} q \\
& -\frac{1}{p} \int_{\mathbb{R}^{N}}\left(\left|u_{1}\right|^{p}+\left|u_{2}\right|^{p}\right) d x \\
& -\frac{2}{p} \int_{\mathbb{R}^{N}}\left|u_{1}\right|^{p / 2}\left|u_{1}\right|^{p / 2} d x \\
\geqslant & \frac{1}{2}\left(\left\|u_{1}\right\|^{2}+\left\|u_{2}\right\|^{2}\right)-\frac{1}{2} q-\frac{\varepsilon}{p}\left(\left\|u_{1}\right\|^{2}+\left\|u_{2}\right\|^{2}\right) \\
& -\frac{C(\varepsilon)}{p}\left(\left|u_{1}\right|_{2}^{2 p(1-\lambda) /(2-\lambda p)}+\left|u_{2}\right|_{2}^{2 p(1-\lambda) /(2-\lambda p)}\right) \\
& -\frac{1}{p} \int_{\mathbb{R}^{N}}\left(\left|u_{1}\right|^{p}+\left|u_{2}\right|^{p}\right) d x \\
\geqslant & \frac{1}{2}\left(\left\|u_{1}\right\|^{2}+\left\|u_{2}\right\|^{2}\right)-\frac{1}{2} q-\frac{2 \varepsilon}{p}\left(\left\|u_{1}\right\|^{2}+\left\|u_{2}\right\|^{2}\right) \\
& -\frac{2 C(\varepsilon)}{p}\left(q_{1}^{p(1-\lambda) /(2-\lambda p)}+q_{2}^{p(1-\lambda) /(2-\lambda p)}\right) .
\end{aligned}
$$

Therefore for sufficiently small $\varepsilon$, we have that

$$
\begin{aligned}
\widetilde{I}\left(u_{1}, u_{2}\right) \geqslant & -\frac{1}{2} q \\
& -C(\varepsilon, p)\left(q_{1}^{p(1-\lambda) /(2-\lambda p)}+q_{2}^{p(1-\lambda) /(2-\lambda p)}\right) \\
> & -\infty .
\end{aligned}
$$

So $-\infty<\widetilde{I}_{q}<0$. $\left(\widetilde{I}_{q}\right)$.

(ii) Let $\left\{\left(u_{1 n}, u_{2 n}\right)\right\}$ be minimizing sequence for problem

By (16), we have that

$$
\begin{aligned}
& \frac{1}{2}\left(\left\|u_{1 n}\right\|^{2}+\left\|u_{2 n}\right\|^{2}\right) \\
& \leqslant \widetilde{I}\left(u_{1 n}, u_{2 n}\right)+\frac{q}{2}+\frac{1}{p} \int_{\mathbb{R}^{N}}\left(\left|u_{1 n}\right|^{p}+\left|u_{2 n}\right|^{p}\right) d x \\
& \quad+\frac{2}{p} \int_{\mathbb{R}^{N}}\left|u_{1}\right|^{p / 2}\left|u_{2}\right|^{p / 2} d x
\end{aligned}
$$

$$
\begin{aligned}
\leqslant & \tilde{I}\left(u_{1 n}, u_{2 n}\right)+\frac{q}{2}+C\left|u_{1 n}\right|_{2}^{p(1-\lambda)}\left\|u_{1 n}\right\|^{p \lambda} \\
& +C\left|u_{2 n}\right|_{2}^{p(1-\lambda)}\left\|u_{2 n}\right\|^{p \lambda} \\
\leqslant & \widetilde{I}\left(u_{1 n}, u_{2 n}\right)+\frac{q}{2} \\
& +C\left(q_{1}^{p(1-\lambda) / 2}\left\|u_{1 n}\right\|^{p \lambda}+q_{2}^{p(1-\lambda) / 2}\left\|u_{2 n}\right\|^{p \lambda}\right) .
\end{aligned}
$$

By $0<\lambda p<2$, we get that $\left\|u_{1 n}\right\|^{2}+\left\|u_{2 n}\right\|^{2}<C$; that is, $\left\{\left(u_{1 n}, u_{2 n}\right)\right\}$ is bounded in $H^{\alpha}\left(\mathbb{R}^{N}\right) \times H^{\alpha}\left(\mathbb{R}^{N}\right)$.

Using an argument by contradiction, we prove the second part of (ii). Assuming $\delta$ does not exist such that $\left|u_{1 n}\right|_{p}+$ $\left|u_{2 n}\right|_{p} \geqslant \delta>0$, then

$$
\lim _{n \rightarrow+\infty} \inf \left(\left|u_{1 n}\right|_{p}+\left|u_{2 n}\right|_{p}\right) \leqslant 0 .
$$

Now we have that

$$
\begin{aligned}
\lim _{n \rightarrow+\infty} \widetilde{I}\left(u_{1 n}, u_{2 n}\right) \\
=\lim _{n \rightarrow+\infty}\left(\int_{\mathbb{R}^{N}}\left(\left|(-\Delta)^{\alpha / 2} u_{1 n}\right|^{2}+\left|(-\Delta)^{\alpha / 2} u_{2 n}\right|^{2}\right) d x\right. \\
-\frac{1}{p} \int_{\mathbb{R}^{N}}\left(\left|u_{1 n}\right|^{p}+\left|u_{2 n}\right|^{p}\right) d x \\
\left.\quad-\frac{2}{p} \int_{\mathbb{R}^{N}}\left|u_{1 n}\right|^{p / 2}\left|u_{2 n}\right|^{p / 2} d x\right) \geqslant-\frac{2}{p} \\
\quad \lim _{n \rightarrow+\infty} \int_{\mathbb{R}^{N}}\left(\left|u_{1 n}\right|^{p}+\left|u_{2 n}\right|^{p}\right) d x \geqslant 0,
\end{aligned}
$$

which contradicts $-\infty<\widetilde{I}_{q}<0$. Therefore for sufficiently large $n$, there exists a positive constant $\delta$ such that $\left|u_{1 n}\right|_{p}+$ $\left|u_{2 n}\right|_{p} \geqslant \delta$. We have completed the proof of the lemma.

Now, for minimizing sequence $\left\{\left(u_{1 n}, u_{2 n}\right)\right\}$ of problem $\left(\widetilde{I}_{q}\right)$ we introduce the Lévy concentration function $Q_{n}(r)=$ $\sup _{y \in \mathbb{R}^{N}} \int_{B(y, r)}\left(\left|u_{1 n}\right|^{2}+\left|u_{2 n}\right|^{2}\right) d x$. Apparently, each function $Q_{n}$ is nondecreasing on $(0,+\infty)$. Using Helly's selection theorem, $\left\{Q_{n}\right\}$ has a convergent subsequence $\left\{Q_{m}\right\}$, such that $\lim _{m \rightarrow+\infty} Q_{m}(r)=Q(r)$, for all $r>0$, where $Q(r)$ is a nondecreasing function. By $0 \leqslant Q_{m}(r) \leqslant q$, there exists $\beta(0 \leqslant \beta \leqslant q)$ satisfying $\lim _{\gamma \rightarrow+\infty} Q(r)=\beta$.

Next, we will prove $\beta=q$.

Lemma 12. Assuming $q>0$ and $\left\{\left(u_{1 n}, u_{2 n}\right)\right\}$ is a minimizing sequence for $\left(\widetilde{I}_{q}\right)$, then $\beta>0$.

Proof. By the definition of $\beta$, we know $\beta \geqslant 0$. Using an argument by contradiction, assume $\beta=0$; then there exist $n_{k}$ and $r_{0}$, satisfying

$$
\lim _{n_{k} \rightarrow+\infty} \sup _{y \in \mathbb{R}^{N}} \int_{B\left(y, r_{0}\right)}\left(\left|u_{1 n_{k}}\right|^{2}+\left|u_{2 n_{k}}\right|^{2}\right) d x \longrightarrow 0 .
$$

By Lemma 5 , we have $\left(u_{1 n_{k}}, u_{2 n_{k}}\right) \rightarrow(0,0)$ in $L^{p}\left(\mathbb{R}^{N}\right) \times$ $L^{p}\left(\mathbb{R}^{N}\right)$. According to Lemma 11(ii), $\left(u_{1 n_{k}}, u_{2 n_{k}}\right) \nrightarrow(0,0)$ 
Discrete Dynamics in Nature and Society

5

in $L^{p}\left(\mathbb{R}^{N}\right) \times L^{p}\left(\mathbb{R}^{N}\right)$. They are contradictory. Therefore, we obtain $\beta>0$.

Lemma 13. For $\gamma_{1}, \gamma_{2}>0$, then $\widetilde{I}_{\gamma_{1}+\gamma_{2}}<\widetilde{I}_{\gamma_{1}}+\widetilde{I}_{\gamma_{2}}$.

Proof. For given $\left(u_{1}, u_{2}\right) \in H^{\alpha}\left(\mathbb{R}^{N}\right) \times H^{\alpha}\left(\mathbb{R}^{N}\right),\left|u_{1}\right|_{2}^{2}+\left|u_{2}\right|_{2}^{2}=$ $\beta>0$, let $u_{i \theta}(x)=\theta^{\gamma_{1}} u_{i}\left(\theta^{\gamma_{2}} x\right)(i=1,2)$, where $\theta>0, \gamma_{1}=$ $2 \alpha /(4 \alpha-(p-2) N), \gamma_{2}=(p-2) /(4 \alpha-(p-2) N)$. Then $\left|u_{1 \theta}\right|_{2}^{2}+\left|u_{2 \theta}\right|_{2}^{2}=\theta \beta$ and

$$
\begin{aligned}
& \widetilde{I}\left(u_{1 \theta}, u_{2 \theta}\right)=\theta^{(2 \alpha p-N(p-2)) /(4 \alpha-(p-2) N)}\left(\frac{1}{2}\right. \\
& \cdot \int_{\mathbb{R}^{N}}\left(\left|(-\Delta)^{\alpha / 2} u_{1}\right|^{2}+\left|(-\Delta)^{\alpha / 2} u_{2}\right|^{2}\right) d x-\frac{1}{p} \\
& \cdot \int_{\mathbb{R}^{N}}\left(\left|u_{1}\right|^{p}+\left|u_{2}\right|^{p}\right) d x-\frac{2}{p} \\
& \left.\quad \cdot \int_{\mathbb{R}^{N}}\left|u_{1}\right|^{p / 2}\left|u_{2}\right|^{p / 2} d x\right),
\end{aligned}
$$

where we apply Lemma 1.

Now, we can derive that

$$
\begin{aligned}
& \tilde{I}_{\theta \beta}=\inf _{\left(u_{1}, u_{2}\right) \in H^{\alpha}\left(\mathbb{R}^{N}\right) \times H^{\alpha}\left(\mathbb{R}^{N}\right),\left|u_{1}\right|_{2}^{2}+\left|u_{2}\right|_{2}^{2}=\theta \beta}\left\{\frac{1}{2}\right. \\
& \cdot \int_{\mathbb{R}^{N}}\left(\left|(-\Delta)^{\alpha / 2} u_{1}\right|^{2}+\left|(-\Delta)^{\alpha / 2} u_{2}\right|^{2}\right) d x-\frac{1}{p} \\
& \cdot \int_{\mathbb{R}^{N}}\left(\left|u_{1}\right|^{p}+\left|u_{2}\right|^{p}\right) d x-\frac{2}{p} \\
& \left.\cdot \int_{\mathbb{R}^{N}}\left|u_{1}\right|^{p / 2}\left|u_{2}\right|^{p / 2} d x\right\}=\theta^{(2 \alpha p-N(p-2)) /(4 \alpha-(p-2) N)} \\
& \cdot \inf _{\left(u_{1}, u_{2}\right) \in H^{\alpha}\left(\mathbb{R}^{N}\right) \times H^{\alpha}\left(\mathbb{R}^{N}\right),\left|u_{1}\right|_{2}^{2}+\left|u_{2}\right|_{2}^{2}=\beta}\left\{\frac{1}{2}\right. \\
& \cdot \int_{\mathbb{R}^{N}}\left(\left|(-\Delta)^{\alpha / 2} u_{1}\right|^{2}+\left|(-\Delta)^{\alpha / 2} u_{2}\right|^{2}\right) d x-\frac{1}{p} \\
& \cdot \int_{\mathbb{R}^{N}}\left(\left|u_{1}\right|^{p}+\left|u_{2}\right|^{p}\right) d x-\frac{2}{p} \\
& \left.\cdot \int_{\mathbb{R}^{N}}\left|u_{1}\right|^{p / 2}\left|u_{2}\right|^{p / 2} d x\right\} \\
& =\theta^{(2 \alpha p-N(p-2)) /(4 \alpha-(p-2) N)} \widetilde{I}_{\beta} .
\end{aligned}
$$

Therefore by Lemma 11(ii) and $(2 \alpha p-N(p-2)) /(4 \alpha-(p-$ 2) N) > 1, we have that

$$
\begin{aligned}
& \widetilde{I}_{\gamma_{1}+\gamma_{2}}=\left(\gamma_{1}+\gamma_{2}\right)^{(2 \alpha p-N(p-2)) /(4 \alpha-(p-2) N)} \widetilde{I}_{1} \\
& \quad<\left(\gamma_{1}^{(2 \alpha p-N(p-2)) /(4 \alpha-(p-2) N)}\right. \\
& \left.\quad+\gamma_{2}^{(2 \alpha p-N(p-2)) /(4 \alpha-(p-2) N)}\right) \widetilde{I}_{1}=\widetilde{I}_{\gamma_{1}}+\widetilde{I}_{\gamma_{2}} .
\end{aligned}
$$

Lemma 14. Assuming $q>0$ and $0<\beta<q$, then $\widetilde{I}_{q} \geqslant \widetilde{I}_{\beta}+\widetilde{I}_{q-\beta}$.

Proof. For given minimizing sequence $\left\{\left(u_{1 n}, u_{2 n}\right)\right\}$ for problem $\left(\widetilde{I}_{q}\right), r>0$, define $Q_{n}(r)=\sup _{y \in \mathbb{R}^{N}} \int_{B(y, r)}\left(\left|u_{1 n}\right|^{2}+\right.$ $\left.\left|u_{2 n}\right|^{2}\right) d x$. Then $Q_{n}$ is nondecreasing on $(0,+\infty)$; moreover for all $r>0,\left\{Q_{n}\right\}$ has subsequences denoted again by $\left\{Q_{n}\right\}$, existing nondecreasing function $Q(r)$ such that $\lim _{n \rightarrow+\infty} Q_{n}(r)=Q(r), \lim _{r \rightarrow+\infty} Q(r)=\beta$. Therefore $\forall \varepsilon>$ $0, \exists r_{\varepsilon}, n_{\varepsilon}$ such that $r>r_{\varepsilon}, n>n_{\varepsilon}$ satisfy

$$
\begin{aligned}
& \beta-\frac{\varepsilon}{2}<Q(r) \leqslant Q(2 r) \leqslant \beta, \\
& \beta-\frac{3 \varepsilon}{4}<Q_{n}(r) \leqslant Q_{n}(2 r) \leqslant \beta+\frac{3 \varepsilon}{4} .
\end{aligned}
$$

Apparently, there exists $\left\{y_{n}\right\} \subset \mathbb{R}^{N}$, satisfying

$$
\begin{gathered}
\int_{B\left(y_{n}, r\right)}\left(\left|u_{1 n}\right|^{2}+\left|u_{2 n}\right|^{2}\right) d x>\beta-\varepsilon, \\
\int_{B\left(y_{n}, 2 r\right)}\left(\left|u_{1 n}\right|^{2}+\left|u_{2 n}\right|^{2}\right) d x<\beta+\varepsilon,
\end{gathered}
$$

where because of definition of $Q_{n}$ and (27).

Setting $v_{i n}=\Psi_{i r}\left(x-y_{n}\right) u_{i n}, w_{i n}=\widetilde{\Psi}_{i r}\left(x-y_{n}\right) u_{i n}, i=1,2$, they satisfy that $\Psi_{i r}(x)=\Psi_{i}(x / r), \widetilde{\Psi}_{i r}(x)=\widetilde{\Psi}_{i}(x / r) ; \forall x \in$ $\widetilde{B}(0,1), \Psi_{i}(x)=1$; for $|x| \geqslant 2, \Psi_{i}(x)=0 ; \Psi_{i}(x) \in C_{0}^{\infty}(B(0,2))$, $\widetilde{\Psi}_{i} \in C^{\infty}\left(\mathbb{R}^{N}\right)$; and $\forall x \in \mathbb{R}^{N}, \Psi_{i}^{2}(x)+\widetilde{\Psi}_{i}^{2}(x)=1$. Then we have that

$$
\begin{gathered}
\beta-\varepsilon<\int_{\mathbb{R}^{N}}\left(\left|v_{1 n}\right|^{2}+\left|v_{2 n}\right|^{2}\right) d x<\beta+\varepsilon, \\
q-\beta-\varepsilon<\int_{\mathbb{R}^{N}}\left(\left|w_{1 n}\right|^{2}+\left|w_{2 n}\right|^{2}\right) d x<q-\beta+\varepsilon .
\end{gathered}
$$

Therefore (29) imply that there exist $\left(\mu_{1 n}, \mu_{2 n}\right)$ and $\left(\nu_{1 n}, v_{2 n}\right)$, $\left|\mu_{\text {in }}-1\right|<\varepsilon,\left|\nu_{\text {in }}-1\right|<\varepsilon, i=1,2$, satisfying $\left|\mu_{1 n} v_{1 n}\right|_{2}^{2}+$ $\left|\mu_{2 n} v_{2 n}\right|_{2}^{2}=\beta,\left|\nu_{1 n} w_{1 n}\right|_{2}^{2}+\left|v_{2 n} w_{2 n}\right|_{2}^{2}=q-\beta$. Then we have that

$$
\begin{gathered}
\widetilde{I}_{\beta} \leqslant \widetilde{I}\left(\mu_{1 n} v_{1 n}, \mu_{2 n} v_{2 n}\right) \leqslant \widetilde{I}\left(v_{1 n}, v_{2 n}\right)+C \varepsilon, \\
\widetilde{I}_{q-\beta} \leqslant \widetilde{I}\left(v_{1 n} w_{1 n}, v_{2 n} w_{2 n}\right) \leqslant \widetilde{I}\left(w_{1 n}, w_{2 n}\right)+C \varepsilon .
\end{gathered}
$$

Using Lemma 7, we derive that

$$
\begin{aligned}
& \left|(-\Delta)^{\alpha / 2}\left(\Psi_{i r}\left(x-y_{n}\right) u_{i n}\right)-\Psi_{i r}\left(x-y_{n}\right)(-\Delta)^{\alpha / 2} u_{i n}\right|_{2} \\
& \quad \leqslant C\left(\left|\nabla \Psi_{i r}\right|_{\infty}\left|(-\Delta)^{(\alpha-1) / 2} u_{i n}\right|_{2}\right. \\
& \left.\quad+\left|(-\Delta)^{\alpha / 2} \Psi_{i r}\left(x-y_{n}\right)\right|_{\tilde{p}}\left|u_{i n}\right|_{p}\right),
\end{aligned}
$$

where $1 / \widetilde{p}+1 / p=1 / 2, i=1,2$. By $\left|\nabla \Psi_{i r}\right|_{\infty}=(1 / r)\left|\nabla \Psi_{i}\right|_{\infty} \leqslant$ $C / r$, the Sobolev inequality, Lemma 11, and (31), we obtain that

$$
\begin{aligned}
& \frac{1}{2} \int_{\mathbb{R}^{N}}\left|(-\Delta)^{\alpha / 2}\left(\Psi_{i r}\left(x-y_{n}\right) u_{i n}\right)\right|^{2} d x \\
& \quad \leqslant \frac{1}{2} \int_{\mathbb{R}^{N}} \Psi_{i r}^{2}\left(x-y_{n}\right)\left|(-\Delta)^{\alpha / 2} u_{i n}\right|^{2} d x+C \varepsilon,
\end{aligned}
$$

$i=1,2$, 
6

Discrete Dynamics in Nature and Society

for sufficiently large $r$. Similarly, for sufficiently large $r$ we have that

$$
\begin{aligned}
& \frac{1}{2} \int_{\mathbb{R}^{N}}\left|(-\Delta)^{\alpha / 2}\left(\widetilde{\Psi}_{i r}\left(x-y_{n}\right) u_{i n}\right)\right|^{2} d x \\
& \quad \leqslant \frac{1}{2} \int_{\mathbb{R}^{N}} \widetilde{\Psi}_{i r}^{2}\left(x-y_{n}\right)\left|(-\Delta)^{\alpha / 2} u_{i n}\right|^{2} d x+C \varepsilon,
\end{aligned}
$$

$$
i=1,2 \text {. }
$$

So we have that

$$
\begin{aligned}
& \frac{1}{2} \int_{\mathbb{R}^{N}}\left(\left|(-\Delta)^{\alpha / 2}\left(\Psi_{1 r}\left(x-y_{n}\right) u_{1 n}\right)\right|^{2}\right. \\
& \left.+\left|(-\Delta)^{\alpha / 2}\left(\Psi_{2 r}\left(x-y_{n}\right) u_{2 n}\right)\right|^{2}\right) d x+\frac{1}{2} \\
& \cdot \int_{\mathbb{R}^{N}}\left(\left|(-\Delta)^{\alpha / 2}\left(\widetilde{\Psi}_{1 r}\left(x-y_{n}\right) u_{1 n}\right)\right|^{2}\right. \\
& \left.+\left|(-\Delta)^{\alpha / 2}\left(\widetilde{\Psi}_{2 r}\left(x-y_{n}\right) u_{2 n}\right)\right|^{2}\right) d x \leqslant \frac{1}{2} \\
& \cdot \int_{\mathbb{R}^{N}}\left(\left|(-\Delta)^{\alpha / 2} u_{1 n}\right|^{2}+\left|(-\Delta)^{\alpha / 2} u_{2 n}\right|^{2}\right) d x \\
& \quad+C \varepsilon .
\end{aligned}
$$

By Lemma 11, (16), (27), $2<p<2+4 \alpha / N$, and the definitions of $\Psi_{i r}, \widetilde{\Psi}_{i r}(i=1,2)$, we can derive that

$$
\begin{aligned}
& -\frac{1}{p} \int_{\mathbb{R}^{N}}\left(\left|\Psi_{1 r}\left(x-y_{n}\right) u_{1 n}\right|^{p}+\left|\Psi_{2 r}\left(x-y_{n}\right) u_{2 n}\right|^{p}\right) d x \\
& +\frac{1}{p} \int_{\mathbb{R}^{N}}\left(\left|u_{1 n}\right|^{p}+\left|u_{2 n}\right|^{p}\right) d x-\frac{1}{p} \\
& \cdot \int_{\mathbb{R}^{N}}\left(\left|\widetilde{\Psi}_{1 r}\left(x-y_{n}\right) u_{1 n}\right|^{p}\right. \\
& \left.+\left|\widetilde{\Psi}_{2 r}\left(x-y_{n}\right) u_{2 n}\right|^{p}\right) d x=-\frac{1}{p} \\
& \cdot \int_{\mathbb{R}^{N}}\left(\left|\Psi_{1 r}\left(x-y_{n}\right) u_{1 n}\right|^{p}\right. \\
& \left.+\left|\Psi_{2 r}\left(x-y_{n}\right) u_{2 n}\right|^{p}\right) d x+\frac{1}{p} \\
& \cdot \int_{\mathbb{R}^{N}}\left(\Psi_{1 r}^{2}\left(x-y_{n}\right)+\widetilde{\Psi}_{1 r}^{2}\left(x-y_{n}\right)\right)\left|u_{1 n}\right|^{p} d x \\
& +\frac{1}{p} \int_{\mathbb{R}^{N}}\left(\Psi_{2 r}^{2}\left(x-y_{n}\right)+\widetilde{\Psi}_{2 r}^{2}\left(x-y_{n}\right)\right)\left|u_{2 n}\right|^{p} d x \\
& -\frac{1}{p} \int_{\mathbb{R}^{N}}\left(\left|\widetilde{\Psi}_{1 r}\left(x-y_{n}\right) u_{1 n}\right|^{p}\right. \\
& \left.+\left|\widetilde{\Psi}_{2 r}\left(x-y_{n}\right) u_{2 n}\right|^{p}\right) d x=\frac{1}{p} \int_{\mathbb{R}^{N}}\left(-\left|\Psi_{1 r}\right|^{p}\right. \\
& \left.+\Psi_{1 r}^{2}\right)\left(x-y_{n}\right)\left|u_{1 n}(x)\right|^{p} d x+\frac{1}{p} \int_{\mathbb{R}^{N}}\left(-\left|\Psi_{2 r}\right|^{p}\right.
\end{aligned}
$$

$$
\begin{aligned}
& \left.+\Psi_{2 r}^{2}\right)\left(x-y_{n}\right)\left|u_{2 n}(x)\right|^{p} d x+\frac{1}{p} \int_{\mathbb{R}^{N}}\left(-\left|\widetilde{\Psi}_{1 r}\right|^{p}\right. \\
& \left.+\widetilde{\Psi}_{1 r}^{2}\right)\left(x-y_{n}\right)\left|u_{1 n}(x)\right|^{p} d x+\frac{1}{p} \int_{\mathbb{R}^{N}}\left(-\left|\widetilde{\Psi}_{2 r}\right|^{p}\right. \\
& \left.+\widetilde{\Psi}_{2 r}^{2}\right)\left(x-y_{n}\right)\left|u_{2 n}(x)\right|^{p} d x \leqslant \frac{4}{p} \int_{\Omega}\left(\left|u_{1 n}(x)\right|^{p}\right. \\
& \left.+\left|u_{2 n}(x)\right|^{p}\right) d x \leqslant \frac{C}{p}\left(\left\|u_{1 n}\right\|_{L^{2}(\Omega)}^{p(1-\lambda)}\right. \\
& \left.\cdot\left\|u_{1 n}\right\|_{W^{\alpha, 2}(\Omega)}^{\lambda p}+\left\|u_{2 n}\right\|_{L^{2}(\Omega)}^{p(1-\lambda)}\left\|u_{2 n}\right\|_{W^{\alpha, 2}(\Omega)}^{\lambda p}\right), \\
& -\int_{\mathbb{R}^{N}}\left|\Psi_{1 r}\left(x-y_{n}\right) u_{1 n}\right|^{p / 2}\left|\Psi_{2 r}\left(x-y_{n}\right) u_{2 n}\right|^{p / 2} d x \\
& -\int_{\mathbb{R}^{N}}\left|\widetilde{\Psi}_{1 r}\left(x-y_{n}\right) u_{1 n}\right|^{p / 2} \\
& \cdot\left|\widetilde{\Psi}_{2 r}\left(x-y_{n}\right) u_{2 n}\right|^{p / 2} d x+\int_{\mathbb{R}^{N}}\left|u_{1 n}\right|^{p / 2} \\
& \cdot\left|u_{2 n}\right|^{p / 2} d x=\int_{\mathbb{R}^{N}}(1 \\
& -\left|\Psi_{1 r}\left(x-y_{n}\right)\right|^{p / 2}\left|\Psi_{2 r}\left(x-y_{n}\right)\right|^{p / 2} \\
& \left.-\left|\widetilde{\Psi}_{1 r}\left(x-y_{n}\right)\right|^{p / 2}\left|\widetilde{\Psi}_{2 r}\left(x-y_{n}\right)\right|^{p / 2}\right)\left|u_{1}\right|^{p / 2} \\
& \cdot\left|u_{2}\right|^{p / 2} d x \leqslant \frac{3}{p} \int_{\Omega}\left|u_{1 n}\right|^{p / 2}\left|u_{2 n}\right|^{p / 2} d x \\
& \leqslant \frac{3}{2 p}\left(\int_{\Omega}\left|u_{1 n}\right|^{p} d x+\int_{\Omega}\left|u_{2 n}\right|^{p} d x\right) \\
& \leqslant \frac{C}{p}\left(\left\|u_{1 n}\right\|_{L^{2}(\Omega)}^{p(1-\lambda)}\left\|u_{1 n}\right\|_{W^{\alpha, 2}(\Omega)}^{\lambda p}+\left\|u_{2 n}\right\|_{L^{2}(\Omega)}^{p(1-\lambda)}\right. \\
& \left.\cdot\left\|u_{2 n}\right\|_{W^{\alpha, 2}(\Omega)}^{\lambda p}\right) \leqslant C \varepsilon
\end{aligned}
$$

where $\Omega=B\left(y_{n}, 2 r\right)-B\left(y_{n}, r\right)$ and $\lambda=N(p-2) / 2 \alpha p$. Therefore by the two inequations, we obtain that

$$
\begin{aligned}
& \widetilde{I}\left(v_{1 n}, v_{2 n}\right)+\widetilde{I}\left(w_{1 n}, w_{2 n}\right) \\
& \leqslant \frac{1}{2} \int_{\mathbb{R}^{N}}\left(\left|(-\Delta)^{\alpha / 2} u_{1 n}\right|^{2}+\left|(-\Delta)^{\alpha / 2} u_{2 n}\right|^{2}\right) d x \\
& \quad-\frac{1}{p} \int_{\mathbb{R}^{N}}\left(\left|u_{1 n}\right|^{p}+\left|u_{2 n}\right|^{p}\right) d x \\
& \quad-\frac{2}{p} \int_{\mathbb{R}^{N}}\left|u_{1 n}\right|^{p / 2}\left|u_{2 n}\right|^{p / 2} d x+C \varepsilon \\
& =\widetilde{I}\left(u_{1 n}, u_{2 n}\right)+C \varepsilon .
\end{aligned}
$$

By (30) and (36), we obtain

$$
\begin{aligned}
\widetilde{I}_{\beta}+\widetilde{I}_{q-\beta}-3 C \varepsilon & \leqslant \widetilde{I}\left(v_{1 n}, v_{2 n}\right)+\widetilde{I}\left(w_{1 n}, w_{2 n}\right)-C \varepsilon \\
& \leqslant \widetilde{I}\left(u_{1 n}, u_{2 n}\right) \longrightarrow \widetilde{I}_{q} .
\end{aligned}
$$

So, we prove that $\widetilde{I}_{q} \geqslant \widetilde{I}_{\beta}+\widetilde{I}_{q-\beta}$. 
Obviously, using Lemmas 11-14, we obtain $\beta=q$.

Proof of Theorem 9. From the above parts; we know that $\beta=q$, that is, there exists a subsequence $\left\{\left(u_{1 m}, u_{2 m}\right)\right\}$ of $\left\{\left(u_{1 n}, u_{2 n}\right)\right\}$ satisfying

$$
\begin{aligned}
& \lim _{r \rightarrow+\infty} \lim _{m \rightarrow+\infty} \sup _{y \in \mathbb{R}^{N}} \int_{B(y, r)}\left(\left|u_{1 m}\right|^{2}+\left|u_{2 m}\right|^{2}\right) d x=\beta \\
& \quad=q .
\end{aligned}
$$

Therefore, for all $k \in \mathbb{N}$, there exist $r_{k}>0, M_{k} \in \mathbb{N}$, such that, $\forall m>M_{k}, \exists y_{m} \in \mathbb{R}^{N}$, having

$$
\int_{B\left(y_{m}, r_{k}\right)}\left(\left|u_{1 m}\right|^{2}+\left|u_{2 m}\right|^{2}\right) d x>q-\frac{1}{k} .
$$

For the above inequality, with a Cantor diagonalization argument, using Lemma 11(ii), $\left|u_{1 m}\right|_{2}^{2}+\left|u_{2 m}\right|_{2}^{2}=q$, we know that there exists $\left(g_{1}, g_{2}\right) \in H^{\alpha}\left(\mathbb{R}^{N}\right) \times H^{\alpha}\left(\mathbb{R}^{N}\right)$ satisfying $\int_{\mathbb{R}^{N}}\left(\left|g_{1}\right|^{2}+\left|g_{2}\right|^{2}\right) d x=q$, such that going if necessary to exist a subsequence $\left\{\left(u_{1 j}\left(\cdot+y_{j}\right), u_{2 j}\left(\cdot+y_{j}\right)\right)\right\}$ of $\left\{\left(u_{1 m}\left(\cdot+y_{m}\right), u_{2 m}(\cdot+\right.\right.$ $\left.\left.\left.y_{m}\right)\right)\right\}$ satisfying

$$
\begin{aligned}
\left(u_{1 j}\left(\cdot+y_{j}\right), u_{2 j}\left(\cdot+y_{j}\right)\right) & \rightarrow\left(g_{1}, g_{2}\right) \\
& \text { in } H^{\alpha}\left(\mathbb{R}^{N}\right) \times H^{\alpha}\left(\mathbb{R}^{N}\right), \\
\left(u_{1 j}\left(\cdot+y_{j}\right), u_{2 j}\left(\cdot+y_{j}\right)\right) & \longrightarrow\left(g_{1}, g_{2}\right) \\
& \text { in } L^{2}\left(\mathbb{R}^{N}\right) \times L^{2}\left(\mathbb{R}^{N}\right) .
\end{aligned}
$$

Then by the interpolation theorem, we obtain that

$$
\begin{aligned}
\left(u_{1 j}\left(\cdot+y_{j}\right), u_{2 j}\left(\cdot+y_{j}\right)\right) & \longrightarrow\left(g_{1}, g_{2}\right) \\
& \text { in } L^{p}\left(\mathbb{R}^{N}\right) \times L^{p}\left(\mathbb{R}^{N}\right) .
\end{aligned}
$$

And by the weak low semicontinuity of the norm in $H^{\alpha}\left(\mathbb{R}^{N}\right)$, we also obtain that

$$
\begin{aligned}
\left(u_{1 j}\left(\cdot+y_{j}\right), u_{2 j}\left(\cdot+y_{j}\right)\right) & \longrightarrow\left(g_{1}, g_{2}\right) \\
& \text { in } H^{\alpha}\left(\mathbb{R}^{N}\right) \times H^{\alpha}\left(\mathbb{R}^{N}\right),
\end{aligned}
$$

and $\left(g_{1}, g_{2}\right)$ is a minimizer of problem $\left(\widetilde{I}_{q}\right)$.

Using an argument by contradiction, we prove

$$
\lim _{n \rightarrow+\infty} \inf _{\left(g_{1}, g_{2}\right) \in \widetilde{G}_{q}}\left(\left\|u_{1 n}-g_{1}\right\|+\left\|u_{2 n}-g_{2}\right\|\right)=0 .
$$

Assume that there exist $\varepsilon_{0}>0$ and a subsequence $\left\{\left(u_{1 n_{j}}, u_{2 n_{j}}\right)\right\}$ of $\left\{\left(u_{1 n}, u_{2 n}\right)\right\}$ satisfying

$$
\inf _{\left(g_{1}, g_{2}\right) \in \widetilde{G}_{q}}\left(\left\|u_{1 n_{j}}-g_{1}\right\|+\left\|u_{2 n_{j}}-g_{2}\right\|\right) \geqslant \varepsilon_{0}>0 .
$$

By the above proof, we know that there exist a subsequence $\left\{\left(u_{1 j}, u_{2 j}\right)\right\}$ of $\left\{\left(u_{1 n_{j}}, u_{2 n_{j}}\right)\right\},\left\{y_{j}\right\} \in \mathbb{R}^{N}$, and $\left(g_{1}, g_{2}\right) \in \widetilde{G}_{q}$ satisfying

$$
\begin{aligned}
\left(u_{1 j}\left(\cdot+y_{j}\right), u_{2 j}\left(\cdot+y_{j}\right)\right) & \longrightarrow\left(g_{1}, g_{2}\right) \\
& \text { in } H^{\alpha}\left(\mathbb{R}^{N}\right) \times H^{\alpha}\left(\mathbb{R}^{N}\right) .
\end{aligned}
$$

So we derive

$$
\begin{aligned}
& \lim _{j \rightarrow+\infty}\left(\left\|u_{1 j}\left(\cdot+y_{j}\right)-g_{1}\right\|+\left\|u_{2 j}\left(\cdot+y_{j}\right)-g_{2}\right\|\right) \\
& \quad=0
\end{aligned}
$$

where since $\left(g_{1}\left(\cdot-y_{j}\right), g_{2}\left(\cdot-y_{j}\right)\right) \in \widetilde{G}_{q}$, we have that

$$
\begin{aligned}
& \lim _{j \rightarrow+\infty} \inf _{\left(g_{1}, g_{2}\right) \in \widetilde{\mathrm{G}}_{q}}\left(\left\|u_{1 j}\left(\cdot+y_{j}\right)-g_{1}\right\|\right. \\
& \left.+\left\|u_{2 j}\left(\cdot+y_{j}\right)-g_{2}\right\|\right)=0 .
\end{aligned}
$$

Then there exists a contradiction. We completed the proof of Theorem 9.

Proof of Theorem 10. Now using an argument by contradiction, we complete the proof of Theorem 10. Assume that the set $\widetilde{G}_{q}$ is not $H^{\alpha}\left(\mathbb{R}^{N}\right) \times H^{\alpha}\left(\mathbb{R}^{N}\right)$-stable. Then there exist $\varepsilon_{0}>$ $0,\left(u_{1 k}^{0}, u_{2 k}^{0}\right) \subset H^{\alpha}\left(\mathbb{R}^{N}\right) \times H^{\alpha}\left(\mathbb{R}^{N}\right)$, and $t_{k} \in[0, T)$ such that

$$
\inf _{\left(g_{1}, g_{2}\right) \in \widetilde{G}_{q}}\left(\left\|u_{1 k}^{0}-g_{1}\right\|+\left\|u_{2 k}^{0}-g_{2}\right\|\right) \leqslant \frac{1}{k}, \quad k \in \mathbb{N},
$$

and for $\left(u_{1 k}, u_{2 k}\right) \in C\left([0, T), H^{\alpha}\left(\mathbb{R}^{N}\right) \times H^{\alpha}\left(\mathbb{R}^{N}\right)\right)$ satisfying (1) with initial value $\left(u_{1 k}^{0}, u_{2 k}^{0}\right)$, we have

$$
\inf _{\left(g_{1}, g_{2}\right) \in \widetilde{G}_{q}}\left(\left\|u_{1 k}\left(t_{k}, \cdot\right)-g_{1}\right\|+\left\|u_{2 k}\left(t_{k}, \cdot\right)-g_{2}\right\|\right) \geqslant \varepsilon_{0} .
$$

By (48), we know that

$$
\begin{aligned}
\lim _{k \rightarrow+\infty} \widetilde{I}\left(u_{1 k}^{0}, u_{2 k}^{0}\right) & =\widetilde{I}_{q}, \\
\lim _{k \rightarrow+\infty}\left|u_{1 k}^{0}\right|_{2}^{2}+\left|u_{2 k}^{0}\right|_{2}^{2} & =q .
\end{aligned}
$$

Then there exist $\left\{\mu_{1 k}\right\},\left\{\mu_{2 k}\right\} \in \mathbb{R}$ satisfying $\lim _{k \rightarrow+\infty} \mu_{1 k}=$ 1 and $\lim _{k \rightarrow+\infty} \mu_{2 k}=1$ such that $\left\{\left(\mu_{1 k} u_{1 k}^{0}, \mu_{2 k} u_{2 k}^{0}\right)\right\}$ is a minimizing sequence of the problem $\left(\widetilde{I}_{q}\right)$. Since $\widetilde{I}\left(u_{1 k}^{0}, u_{2 k}^{0}\right)=$ $\widetilde{I}\left(u_{1 k}\left(t_{k}\right), u_{2 k}\left(t_{k}\right)\right),\left|u_{1 k}^{0}\right|_{2}^{2}=\left|u_{1 k}\left(t_{k}\right)\right|_{2}^{2}$ and $\left|u_{2 k}^{0}\right|_{2}^{2}=\left|u_{2 k}\left(t_{k}\right)\right|_{2}^{2}$, we easily derive that $\left\{\left(\mu_{1 k} u_{1 k}\left(t_{m}\right), \mu_{2 k} u_{2 k}\left(t_{m}\right)\right)\right\}$ is a minimizing sequence of the problem $\left(\widetilde{I}_{q}\right)$. By Theorem 9, there exists $\left\{\left(g_{1 k_{m}}, g_{2 k_{m}}\right)\right\} \subset \widetilde{G}_{q}$, for sufficiently large $k_{m}$ satisfying

$$
\begin{aligned}
& \left\|\mu_{1 k_{m}} u_{1 k_{m}}\left(t_{k_{m}}\right)-g_{1 k_{m}}\right\|+\left\|\mu_{2 k_{m}} u_{2 k_{m}}\left(t_{k_{m}}\right)-g_{2 k_{m}}\right\| \\
& \quad<\frac{\varepsilon_{0}}{2} .
\end{aligned}
$$

Therefore by Lemma 11(ii), (49), $\lim _{k \rightarrow+\infty} \mu_{1 k}=1$, $\lim _{k \rightarrow+\infty} \mu_{2 k}=1$, and the above inequality, for sufficiently large $k_{m}$, we derive that

$$
\begin{aligned}
\varepsilon_{0} \leqslant & \left\|u_{1 k_{m}}\left(t_{k_{m}}\right)-g_{1 k_{m}}\right\|+\left\|u_{2 k_{m}}\left(t_{k_{m}}\right)-g_{2 k_{m}}\right\| \\
\leqslant & \left\|u_{1 k_{m}}\left(t_{k_{m}}\right)-\mu_{1 k_{m}} u_{1 k_{m}}\left(t_{k_{m}}\right)\right\| \\
& +\left\|\mu_{1 k_{m}} u_{1 k_{m}}\left(t_{k_{m}}\right)-g_{1 k_{m}}\right\| \\
& +\left\|u_{2 k_{m}}\left(t_{k_{m}}\right)-\mu_{2 k_{m}} u_{2 k_{m}}\left(t_{k_{m}}\right)\right\| \\
& +\left\|\mu_{2 k_{m}} u_{2 k_{m}}\left(t_{k_{m}}\right)-g_{2 k_{m}}\right\| \leqslant \frac{3 \varepsilon_{0}}{4} .
\end{aligned}
$$


Then obtaining a contradiction, the set $\widetilde{G}_{q}$ is $H^{\alpha}\left(\mathbb{R}^{N}\right) \times$ $H^{\alpha}\left(\mathbb{R}^{N}\right)$-stable with respect to (1). The proof is completed.

\section{Conflict of Interests}

The authors declare that there is no conflict of interests regarding the publication of this paper.

\section{Acknowledgments}

This work was supported by the NSF of China (nos. $11371183,11271050)$ and the NSF of Shandong Province (no. ZR2013AM004).

\section{References}

[1] R. Hilfer, Application of Fractional Calculus in Physics, World Scientific, Singapore, 2000.

[2] A. Kilbas, H. M. Srivastava, and J. J. Trujillo, Theory and Applications of Fractional Differential Equations, vol. 204 of North-Holland Mathematics Studies, Elsevier, 2006.

[3] R. Metzler and J. Klafter, "The random walk's guide to anomalous diffusion: a fractional dynamics approach," Physics Reports, vol. 339, no. 1, pp. 1-77, 2000.

[4] R. Metzler and J. Klafter, "The restaurant at the end of the random walk: recent developments in the description of anomalous transport by fractional dynamics," Journal of Physics A: Mathematical and General, vol. 37, no. 31, pp. R161-R208, 2004.

[5] K. S. Miller and B. Ross, An Introduction to the Fractional Calculus and Fractional Differential Equations, Wiley-Interscience, New York, NY, USA, 1993.

[6] I. Podlubny, Fractional Differential Equations, vol. 198 of Mathematics in Science and Engineering, Academic Press, San Diego, Calif, USA, 1999.

[7] I. Podlubny, Fractional Differential Equations, vol. 198 of Mathematics in Science and Engineering, Elsevier, London, UK, 1999.

[8] R. P. Feynman and A. R. Hibbs, Quantum Mechanics and Path Integrals, McGraw-Hill, New York, NY, USA, 1965.

[9] N. Laskin, "Fractional quantum mechanics and Lévy path integrals," Physics Letters. A, vol. 268, no. 4-6, pp. 298-305, 2000.

[10] N. Laskin, "Fractional quantum mechanics," Physical Review E, vol. 62 , no. 3, pp. 3135-3145, 2000.

[11] N. Laskin, "Fractional Schrödinger equations," Physical Review E, vol. 66, no. 5, pp. 56-108, 2002.

[12] X. Guo and M. Xu, "Some physical applications fractional Schrödinger equation," Journal of Mathematical Physics, vol. 47, pp. 82-104, 2006.

[13] R. Herrmann, "Gauge invariance in fractional field theories," Physics Letters A, vol. 372, no. 34, pp. 5515-5522, 2008.

[14] P. Rozmej and B. Bandrowski, "On fractional Schrödinger equation," Computational Methods in Science and Technology, vol. 16, no. 2, pp. 191-194, 2010.

[15] H. Berestycki and P.-L. Lions, "Nonlinear scalar field equations, I existence of a ground state," Archive for Rational Mechanics and Analysis, vol. 82, no. 4, pp. 313-345, 1983.

[16] H. Berestycki and P.-L. Lions, "Nonlinear scalar field equations, II existence of infinitely many solutions," Archive for Rational Mechanics and Analysis, vol. 82, no. 4, pp. 347-375, 1983.
[17] T. Cazenave and P.-L. Lions, "Orbital stability of standing waves for some nonlinear Schrödinger equations," Communications in Mathematical Physics, vol. 85, no. 4, pp. 549-561, 1982.

[18] V. E. Zakharov and A. B. Shabat, "Exact theory of two-dimensional self-focusing and one-dimensional self-modulation of waves in nonlinear media," Soviet Journal of Experimental and Theoretical Physics, vol. 34, pp. 62-69, 1972.

[19] X. Song, "Stability and instability of standing waves to a system of Schrödinger equations with combined power-type nonlinearities," Journal of Mathematical Analysis and Applications, vol. 366, no. 1, pp. 345-359, 2010.

[20] F. Genoud, "Existence and orbital stability of standing waves for some nonlinear Schrödinger equations, perturbation of a model case," Journal of Differential Equations, vol. 246, no. 5, pp. 19211943, 2009.

[21] B. Guo and D. W. Huang, "Existence and stability of standing waves for nonlinear fractional Schrödinger equations," Journal of Mathematical Physics, vol. 53, no. 8, Article ID 083702, 2012.

[22] D. Wu, "Existence and stability of standing waves for nonlinear fractional Schrödinger equations with Hartree type nonlinearity," Journal of Mathematical Analysis and Applications, vol. 411, no. 2, pp. 530-542, 2014.

[23] J. Hu, J. Xin, and H. Lu, "The global solution for a class of systems of fractional nonlinear Schrödinger equations with periodic boundary condition," Computers and Mathematics with Applications, vol. 62, no. 3, pp. 1510-1521, 2011.

[24] R. A. Adams, Sobolev Spaces, Academic Press, New York, NY, USA, 1975. 




Advances in

Operations Research

mansans

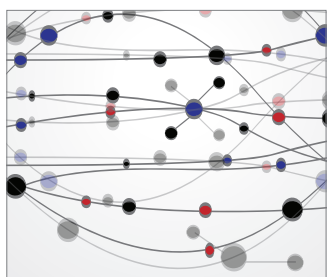

The Scientific World Journal
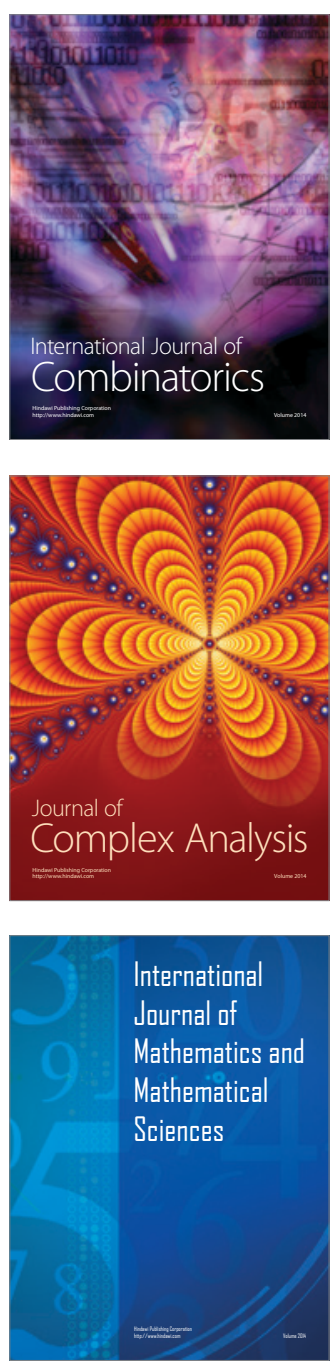
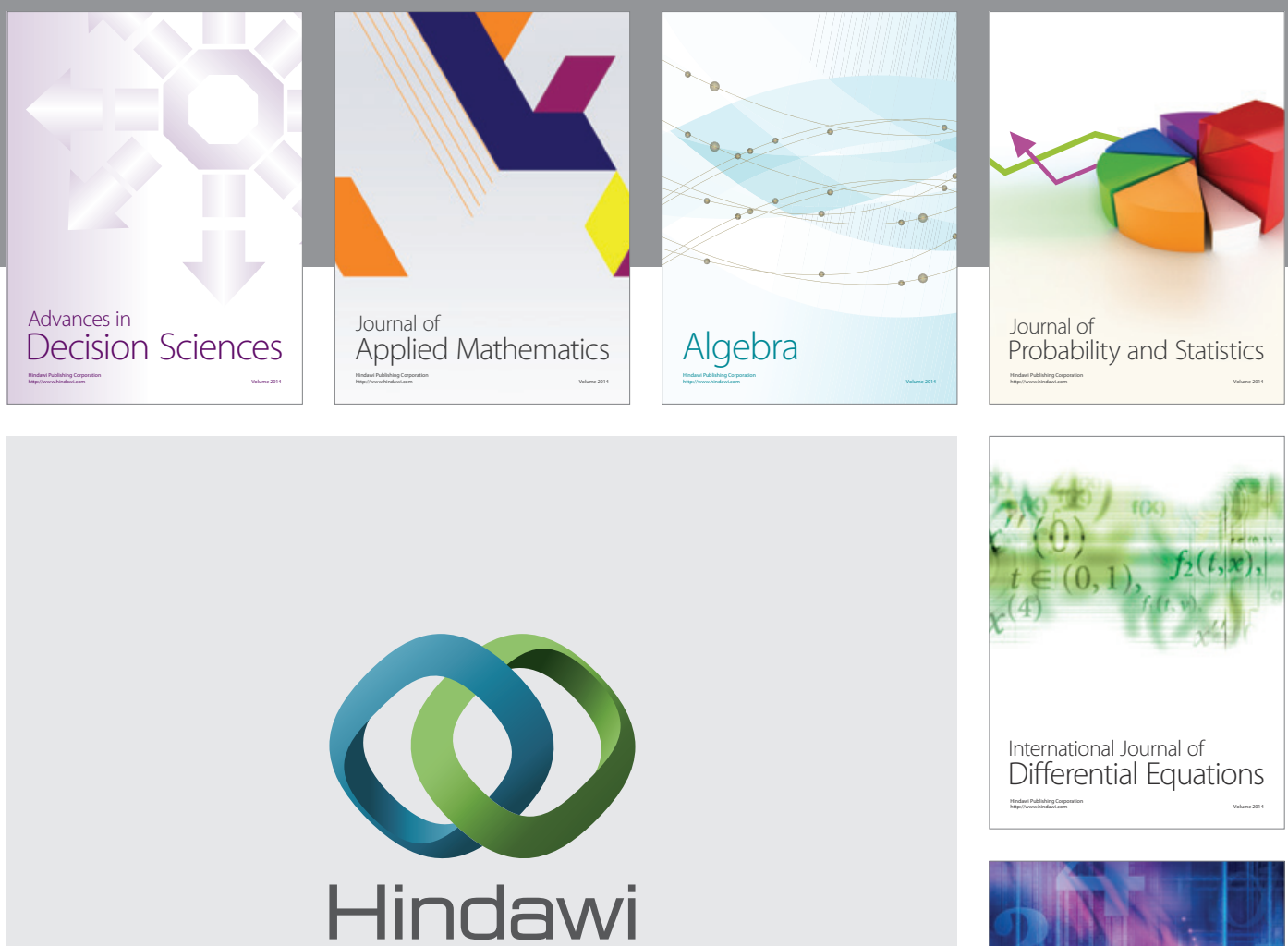

Submit your manuscripts at http://www.hindawi.com
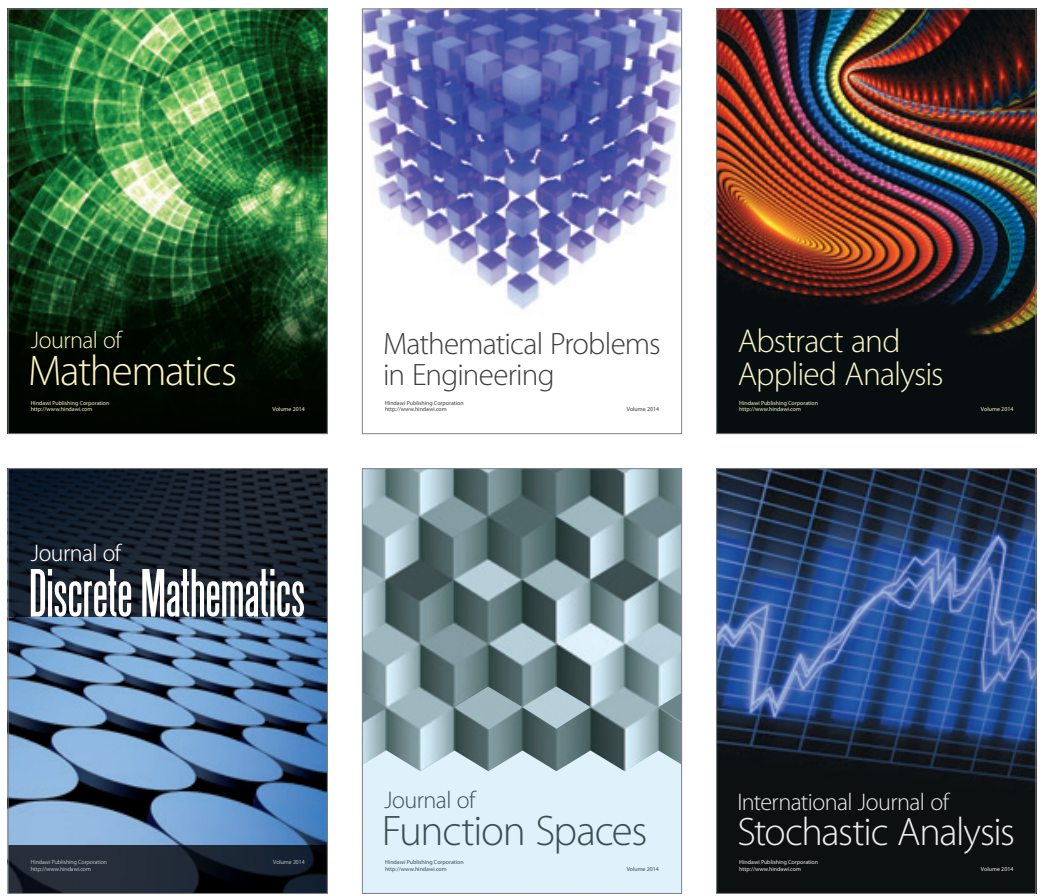

Journal of

Function Spaces

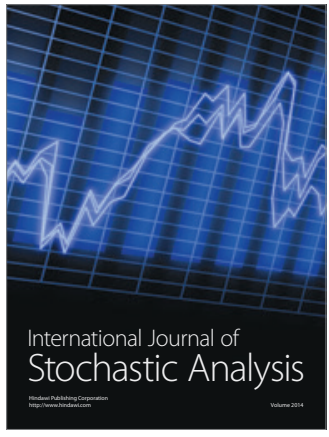

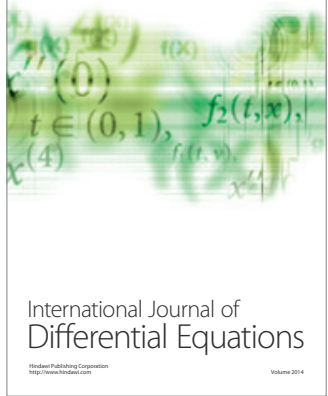
